\title{
Effects of pentobarbital on upper airway patency during sleep
}

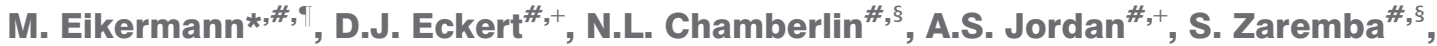 \\ S. Smith ${ }^{\#,+}$, C. Rosow* ${ }^{\star}$, and A. Malhotra ${ }^{\#,+}$
}

ABSTRACT: We hypothesised that pentobarbital would improve upper airway mechanics based on an increase in latency to arousal and amplitude of the phasic genioglossus electromyogram (EMG), and a decrease in the active upper airway critical closing pressure (Pcrit).

12 healthy subjects received pentobarbital $(100 \mathrm{mg})$ or placebo in a double-blind, crossover protocol. During wakefulness, we measured the genioglossus reflex response to negative pressure pulses. During sleep, carbon dioxide was insufflated into the inspired air. Airway pressure was then decreased in a stepwise fashion until arousal from sleep.

With basal breathing during sleep: flow rate was lower in volunteers given pentobarbital; endtidal $\mathrm{CO}_{2}$ concentration and upper airway resistance were greater; and $P$ crit was unaffected (pentobarbital mean \pm SD $-11.7 \pm 4.5$ versus placebo $-10.25 \pm 3.6 \mathrm{cmH}_{2} \mathrm{O} ; \mathrm{p}=0.11$ ). Pentobarbital increased the time to arousal (297 $\pm 63 \mathrm{~s}$ versus $232 \pm 67 \mathrm{~s} ; \mathrm{p}<0.05)$, at which time phasic genioglossus EMG was higher $(6.2 \pm 4.8 \%$ maximal versus $3.1 \pm 3 \% ; p<0.05)$ as were $\mathrm{CO}_{2}$ levels. The increase in genioglossus EMG after $\mathrm{CO}_{2}$ administration was greater after pentobarbital versus placebo. Pentobarbital did not affect the genioglossus negative-pressure reflex.

Pentobarbital increases the time to arousal and stimulates genioglossus muscle activity, but it also increases upper airway resistance during sleep.

KEYWORDS: Airway, arousal threshold, lung, obstructive sleep apnoea/hypopnoea syndrome, sleep-disordered breathing

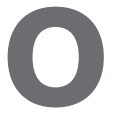

bstructive sleep apnoea (OSA) is a common disorder [1], characterised by repetitive pharyngeal collapse during sleep [2]. Arousal from sleep is traditionally believed to be an important mechanism for reestablishing airway patency in OSA. However, recent data suggest that an excessively low arousal threshold may predispose an individual to recurrent arousals, and the hyperventilation that occurs following arousal may produce hypocapnia during subsequent sleep [3].

Reduced carbon dioxide values may lead to either central or obstructive apnoea, depending on the prevailing upper airway mechanics $[4,5]$. Thus, a low arousal threshold may contribute to sleep apnoea, at least in some individuals $[3,6,7]$. Premature arousal during an obstructive event may prevent adequate upper airway muscle recruitment because there is inadequate accumulation of respiratory stimuli (i.e. $\mathrm{CO}_{2}$ and negative intrapharyngeal pressure). Recent data suggest that treatment with certain hypnotics may not be deleterious and may even improve this condition in certain patients [8-11]. A number of medications have been demonstrated to raise the arousal threshold, and recent data suggest that treatment with some hypnotics and antidepressants may improve OSA manifestations [8-11]. Triazolam and ethanol have been shown to increase the arousal threshold in response to airway occlusion in normal subjects $[12,13]$, and the antidepressants mirtazipine and trazodone may improve manifestations of sleep apnoea [8]. It has been shown that trazodone co-administered with Ltryptophan can treat sleep-disordered breathing in an animal model of OSA [14]. In humans, trazodone may improve airway mechanics by raising the arousal threshold [10] and potentially allowing both negative airway pressure and $\mathrm{CO}_{2}$ to increase, thereby activating pharyngeal dilator muscles. The upper airway dilator muscles (e.g. genioglossus) are known to respond during sleep to combinations of negative pressure and hypercapnia better than to either stimulus alone [15].

Recent data show that pentobarbital can increase genioglossus phasic activity in the rat $[11,16]$. However, pentobarbital also produces some less desirable effects on airway physiology in the rat: it causes a dose-dependent reduction in both diaphragmatic activity and tonic (expiratory)

\section{AFFILIATIONS}

*Dept of Anesthesia, Massachusetts General Hospital,

\#Harvard Medical School,

${ }^{+}$Divisions of Sleep Medicine and Pulmonary/Critical Care, Brigham and Women's Hospital, and

${ }^{\S}$ Dept of Neurology, Beth Israel Deaconess Medical Center, Boston, MA, USA.

-Universitätsklinikum Essen, Klinik fuer Anästhesie und Intensivmedizin, Essen, Germany.

\section{CORRESPONDENCE}

M. Eikermann

Dept of Anesthesia and Critical Care Massachusetts General Hospital

55 Fruit St

Boston, MA 02114-2696

USA

E-mail: meikermann@partners.org

Received:

Sept 292009

Accepted after revision:

Dec 182009

First published online:

Dec 232009 
genioglossus activity [16]. Large doses of pentobarbital can also impair the genioglossus negative-pressure reflex (i.e. reflex activation in response to a sudden decrease in pressure) [16], which may adversely affect upper airway patency.

Based on preclinical data, we hypothesised that pentobarbital would delay arousal in human subjects following a standardised negative-pressure stimulus and that this delay would augment genioglossus muscle activity and improve upper airway closing pressure. To test these hypotheses, we performed a randomised, double-blind, placebo-controlled, crossover study comparing $100 \mathrm{mg}$ pentobarbital to placebo.

\section{MATERIALS AND METHODS}

The protocol was approved by the Institutional Review Board of Brigham and Women's Hospital (Boston, MA, USA). 12 healthy adult volunteers (ages 18-48 yrs; body mass index $<25 \mathrm{~kg} \cdot \mathrm{m}^{-2}$ ) were recruited to participate in the study. We excluded people with concurrent cardiopulmonary disease, including untreated hypertension, kidney disease, liver disease, neuromuscular disease, sleep disorders and psychiatric disease. We also excluded those taking medication known to affect sleep, upper airway muscles or respiratory function (e.g. oral contraceptives, hormone replacement therapy, theophylline, acetazolamide, stimulants, sedatives, thyroxine and antidepressants). Finally, we excluded those with a history of lidocaine or barbiturate allergy, or acute intermittent porphyria. Subjects were recruited through posted flyers, e-mail and newspaper advertisements.

\section{Protocol}

Subjects were studied twice, once with pentobarbital $100 \mathrm{mg}$ (diluted in cherry syrup) and once with placebo treatment (cherry syrup alone) in a randomised, double-blind fashion, with $\geqslant 10$ days between treatments (fig. 1).

On study days, subjects were admitted into our Clinical Research Center (Beth Israel Deaconess Medical Center, Boston, MA, USA) at approximately 20:00 h. Pre-menopausal females underwent a urinary pregnancy test prior to medication administration. After the study procedures were explained, adhesive surface electrodes were attached to the scalp (electroencephalogram (EEG)), face (electrooculogram), and chin (electromyogram (EMG)). Following this, both nostrils were decongested (oxymetazoline $\mathrm{HCl}$ ), and one nostril and the back of the throat were anaesthetised with $\sim 0.5 \mathrm{~mL}$ of topical $4 \%$ lidocaine (20-40 mg). Airway pressure was monitored at the level of the epiglottis (epiglottic pressure) using a pressure-tipped Millar catheter that was inserted through the anaesthetised nostril and secured with tape. Three surface electrocardiogram electrodes were placed on the chest and shoulders.

The area under the tongue (3-4 mm lateral to the frenulum on each side) was topically anaesthetised with lidocaine for insertion of genioglossus muscle electrodes. Two needles (25gauge) containing 30-gauge stainless steel recording electrodes were inserted into the genioglossus muscle. The needles were then quickly removed leaving the recording electrodes in place. Recordings were bipolar with a forehead ground. The subject was instructed to perform several manoeuvres to determine maximal activity of the genioglossus muscle (maximal tongue protrusion, swallowing, negative inspiratory force).

A nasal continuous positive airway pressure (CPAP) mask placed over the subject's nose and held in place with a head strap permitted measurement of breathing rate, inspired volume (integrated inspiratory flow signal from a pneumotachograph), mask pressure and $\mathrm{CO}_{2}$ levels. An arterial oxygen saturation probe was attached to one of the subject's fingers or earlobes to monitor oxygenation.

Before and $60 \mathrm{~min}$ after administration of the study drug, baseline data were collected during a 10-min period of normal breathing while the subject was awake (fig. 1). In addition, $\sim 40$ brief pulses of negative airway pressure $(200 \mathrm{~ms})$ were delivered during early inspiration every two to eight breaths to measure the genioglossus negative pressure reflex as described previously [17, 18].

Subjects were then allowed to fall asleep while breathing room air at atmospheric pressure. When breathing was stable for a period of $5 \mathrm{~min}$, the respiratory response to $\mathrm{CO}_{2}$ was assessed. Sufficient $\mathrm{CO}_{2}$ (10\% balanced with nitrogen) was added to the inspired air to produce stable elevations of end-tidal $\mathrm{CO}_{2}$ that were first 5, then $10 \mathrm{mmHg}$ higher than baseline.

Subjects were then awoken and placed on $3 \mathrm{cmH}_{2} \mathrm{O}$ CPAP. Once they had reached stable nonrapid eye movement sleep again, we increased the CPAP level to alleviate any degree of flow limitation. When steady state stage-II sleep without flow

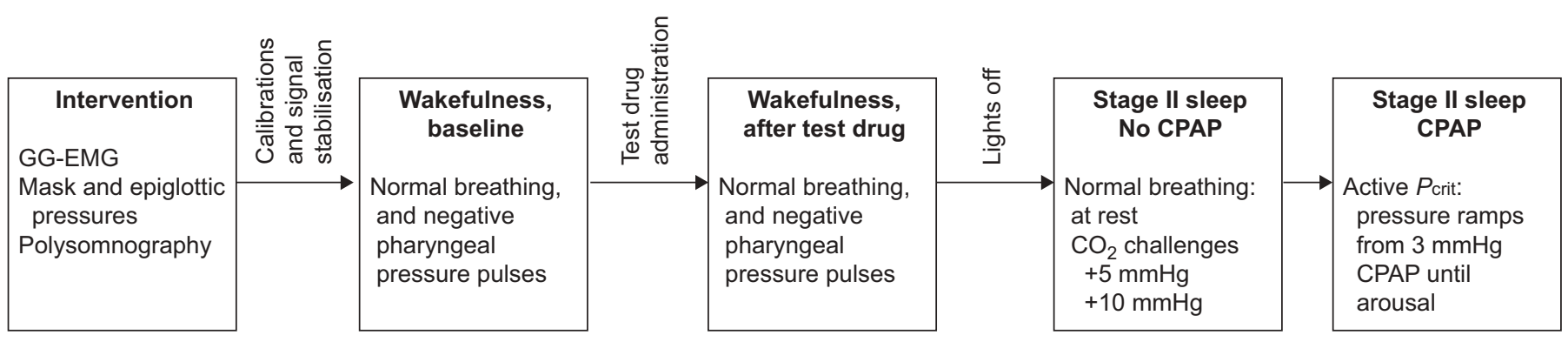

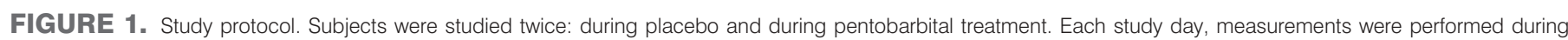

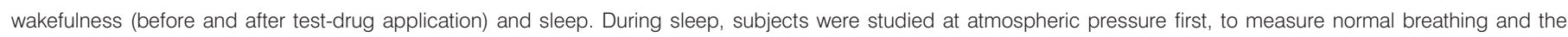

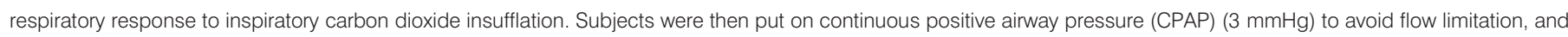
negative-pressure ramps were performed until arousal. GG-EMG: genioglossus electromyography; $P$ crit: critical closing pressure. 
limitation was achieved, we reduced airway opening pressure in 2- $\mathrm{cmH}_{2} \mathrm{O}$ steps, and subjects were monitored for arousal (i.e. presence of $\alpha$ wave activity on the EEG). If the subject did not have an arousal for $1 \mathrm{~min}$ we proceeded to the next step. Continuous negative airway pressure was used if subatmospheric pressures were required. This titration procedure (hereafter called a negative pressure ramp) was repeated up to a maximum of 15 times throughout the night. Following data collection, all equipment was removed and the subjects were allowed to recover in the General Clinical Research Center for $\geqslant 8 \mathrm{~h}$ after drug ingestion and until they felt alert enough to go home. Discharge readiness was confirmed by a licensed physician who was not involved in the study.

\section{Data analysis}

A single experienced, registered sleep technician, blinded to the experimental manipulations, defined the presence of arousal and sleep stage according to standard criteria [19]. For analysis of arousability, we have only included data in the analysis that occurred $>30 \mathrm{~s}$ after onset of a pressure reduction.

The effect of pentobarbital and placebo on the excitation component of the genioglossus negative pressure reflex was compared within subjects pre versus post administration according to methods that have been previously described $[17,18]$. Analysis was performed blinded to the study condition. Briefly, the genioglossus (GG)-EMG signal was rectified and averaged for all negative pressure pulses that were free from movement and swallow artefact. The amplitude of the initial GG-EMG peak was expressed as a percentage of the baseline activity. Reflex latency was defined as the time to peak GG-EMG from time zero (the last point preceding the sudden decrement in the ensemble-averaged pressure signal).

Wakefulness, phasic and tonic GG-EMG, airflow, upper airway resistance and end-tidal $\mathrm{CO}_{2}$ were measured during quiet breathing before and $60 \mathrm{~min}$ after study drug. Maximal genioglossus activation manoeuvres allowed an EMG scale to be created for each subject between electrical 0 and the single highest value encountered (100\%) [20]. During sleep, GG-EMG just prior to arousal was calculated by averaging the value during three breaths immediately before arousal.

A standardised protocol for assessing the active Pcrit was implemented as previously described (fig. 2) [21]. When inspiratory flow limitation was stable, nasal pressure and maximum inspiratory flow were obtained from three breaths at the end of a 2-min period of stable stage 2 sleep. Flow limitation was defined as: unchanged inspiratory flow despite a further decrease in pharyngeal (epiglottic) pressure. Mask pressure was then plotted versus maximum flow for the flow limited breaths and fitted using a linear regression model.

Time to arousal was defined as the time from onset of the negative pressure ramp to an arousal as detected by EEG $\alpha$ wave activity.

Upper airway resistance (epiglottic catheter to mask) was measured at a flow of $0.2 \mathrm{~L} \cdot \mathrm{s}^{-1}$, which is generally on the linear portion of the pressure/flow curve.

\section{Statistical analysis}

The primary dependent variable was time to arousal. We also tested the hypothesis that time to arousal is significantly longer in subjects following pentobarbital $100 \mathrm{mg}$ compared with placebo. We tested the secondary hypothesis that phasic GGEMG just prior to arousal would be significantly higher in volunteers given pentobarbital compared with placebo. Finally, we tested the exploratory hypothesis that active upper airway closing pressure would be lower (more negative) in volunteers given pentobarbital versus placebo.

Based on the observations of BERRY and co-workers [12, 13] who observed a longer time to arousal from sleep in volunteers given alcohol and triazolam, we anticipated a 30\% difference and a SD of $10 \%$. Based on the data of YOUNES et al. [11], who observed a higher phasic genioglossus activity at the time of arousal in rats given pentobarbital compared with placebo, we expected a $50 \%$ difference between groups in phasic genioglossus activity (SD 10\%). Finally, based on the association of phasic genioglossus activity and upper airway closing pressure in humans, we expected a 10\% difference (SD 10\%) in Pcrit between groups [22]. We calculated that a total sample size of 10 volunteers would provide sufficient power to detect a significant difference in the primary and secondary hypotheses (power $=0.8, \alpha<0.05$ ). Paired $\mathrm{t}$-tests were used for testing the main hypotheses. We used a general linear model (mixed model) to analyse the GG-EMG response to $\mathrm{CO}_{2}$. We used GG-EMG as the dependent variable and drug (pentobarbital versus placebo), respiratory phase (phasic versus tonic), and $\mathrm{CO}_{2}$ level (baseline, $+5 \mathrm{mmHg}$ and $+10 \mathrm{mmHg}$ ) as independent variables.

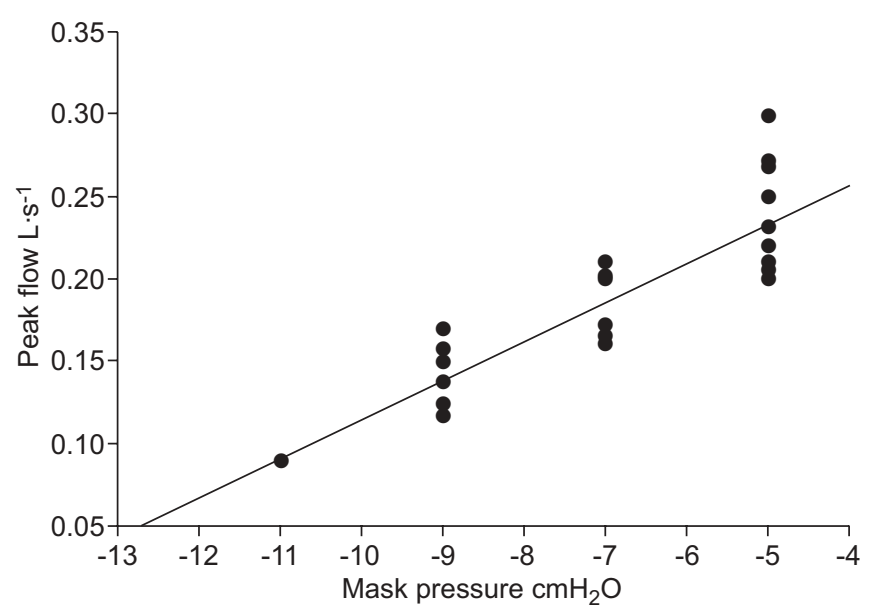

FIGURE 2. Method of calculation of upper airway critical closing pressure $\left(P_{\text {crit }}\right)$ during stage II sleep by linear regression in one volunteer. Peak air flow during flow-limited breathing is plotted as a function of mask pressure. Throughout the study night, 12 negative-pressure ramps were performed, while flow limitation was observed. Values derived from 31 flow-limited breaths were used for analysis and extrapolated to $P$ crit (mask pressure at zero flow) by linear regression. Note that, at a given mask pressure, peak flow during flow-limited breathing varied throughout the overnight study, suggesting that the balance between the collapsing and dilating forces acting at the upper airway varied throughout the night. 
The results are expressed as the mean $\pm \mathrm{SD}$, unless otherwise indicated. SPSS Version 11.0 and Sigma Stat Version 3.0 (both SPSS, Inc., Chicago, IL, USA) were used for statistical analysis.

\section{RESULTS}

One volunteer was excluded during the first study night due to inability to sleep, leaving data from 11 volunteers (three males and eight females) aged $35 \pm 10$ yrs (height $173 \pm 8 \mathrm{~cm}$, weight $67 \pm 8 \mathrm{~kg}$ ) for analysis.

\section{Effects of pentobarbital during wakefulness}

During wakefulness, pentobarbital did not affect breathing or genioglossus muscle function. There was no significant difference in minute ventilation, tidal volume $(V \mathrm{~T})$, end-tidal $\mathrm{CO}_{2}$, duty cycle (time taken for inspiration $(t \mathrm{I}) /$ total time of respiratory cycle $(t$ tot $))$, flow-rate $(V \mathrm{~T} / \mathrm{t})$, phasic and tonic GGEMG (table 1).

Negative pressure reflex activation of the GG-EMG was robust with pentobarbital and placebo (more than two-fold increase, table 2). Amplitude and latency of the genioglossus reflex activation did not differ before and after pentobarbital administration. Similarly, reflex properties did not differ before versus after placebo.

\section{Effects of pentobarbital during sleep}

Respiratory function during normal breathing

During normal stage II sleep (atmospheric mask pressure), flow rate was significantly lower in volunteers given pentobarbital, while end-tidal $\mathrm{CO}_{2}$ concentration, and upper airway resistance were significantly greater compared with both baseline (same study day) and placebo. Duty cycle was significantly greater after pentobarbital compared with baseline (table 2). VT and respiratory frequency did not differ between treatments.

\section{Responses to pressure drops}

Time to respiratory-induced arousal from stage II sleep

For each subject, we decreased CPAP an average of $10 \pm 3$ times during stage II sleep. Onset of flow limitation occurred at $-3.6 \pm 2.5$ versus $-3.4 \pm 3.2 \mathrm{cmH}_{2} \mathrm{O}$ in the placebo and pentobarbital night, respectively, without differences between groups $(\mathrm{p}=0.8)$.

There was no difference in the number of pressure drops prior to arousal between placebo and pentobarbital trials. However, arousal from stable stage II sleep occurred significantly later with pentobarbital $(297 \pm 63$ versus $232 \pm 67$ s after stimulus; $\mathrm{p}<0.05)$, and mask pressure was therefore lower $(-2.9 \pm 3.2$ versus $\left.-0.5 \pm 2.3 \mathrm{cmH}_{2} \mathrm{O} ; \mathrm{p}<0.05\right)$.

\section{Genioglossus function and upper airway pressure flow} relationship just prior to arousal from sleep

Phasic genioglossus activity during flow-limited breathing just prior to arousal was significantly higher after pentobarbital compared with placebo (fig. 3). End-tidal $\mathrm{CO}_{2}$ concentration (first breath after termination of pressure drop) was modestly but significantly higher, with pentobarbital versus placebo $(45.6 \pm 4.6$ versus $42 \pm 1.1 \mathrm{mmHg}$; $\mathrm{p}<0.05)$.

The range of mask pressure values used for assessment of Pcrit was 1- $-17 \mathrm{cmH}_{2} \mathrm{O}$. The change in Pcrit (pentobarbital $-1.7 \pm 4.5$ versus placebo $-10.25 \pm 3.6 \mathrm{cmH}_{2} \mathrm{O} ; \mathrm{p}=0.11$; fig. 4) and the increase in tonic genioglossus activity with pentobarbital compared with placebo did not reach statistical significance $(p=0.082)$. In assessing whether genioglossus activation was mechanically effective, we found that the rise in tonic (but not phasic) GG-EMG was predictive of the improvement in airway mechanics (i.e. active $P$ crit $)(r=-0.66$; $\mathrm{p}=0.03$; fig. 5).

\section{TABLE 1 Respiratory function during normal breathing}

\begin{tabular}{|c|c|c|c|c|c|c|}
\hline & \multicolumn{4}{|c|}{ Wakefulness } & \multicolumn{2}{|c|}{ Sleep } \\
\hline & \multicolumn{2}{|c|}{$\begin{array}{c}\text { Before test drug application } \\
\text { (baseline) }\end{array}$} & \multicolumn{2}{|c|}{ After test drug application } & \multicolumn{2}{|c|}{ During stage II sleep } \\
\hline & Pentobarbital & Placebo & Pentobarbital & Placebo & Pentobarbital & Placebo \\
\hline Upper airway resistance $\mathrm{CMH}_{2} \mathrm{O} \cdot \mathrm{L}^{-1} \cdot \mathrm{S}$ & $2.9 \pm 4.4$ & $2.3 \pm 3.4$ & $5.2 \pm 4.8$ & $1.6 \pm 0.9$ & $7.1 \pm 8.3^{*,+}$ & $2.5 \pm 2.6$ \\
\hline End-tidal $\mathrm{CO}_{2} \mathrm{mmHg}$ & $41.6 \pm 2.7$ & $40.1 \pm 2.6$ & $42.4 \pm 3.2$ & $40.9 \pm 1.9$ & $44.5 \pm 3.4^{*,+}$ & $42.3 \pm 2.3$ \\
\hline$V^{\prime} E L \cdot \min ^{-1}$ & $6.8 \pm 1.9$ & $6.9 \pm 1.2$ & $7.0 \pm 1.5$ & $6.8 \pm 1.5$ & $6.6 \pm 1.7$ & $6.4 \pm 1.1$ \\
\hline$V_{T} L \cdot \min ^{-1}$ & $0.40 \pm 0.15$ & $0.37 \pm 0.06$ & $0.37 \pm 0.09$ & $0.38 \pm 0.07$ & $0.42 \pm 0.15$ & $0.40 \pm 0.11$ \\
\hline Duty cycle $\#$ & $0.43 \pm 0.03$ & $0.41 \pm 0.03$ & $0.46 \pm 0.06$ & $0.43 \pm 0.09$ & $0.47 \pm 0.07^{+}$ & $0.44 \pm 0.09$ \\
\hline Flow rate $\mathrm{mL} \cdot \mathrm{s}^{-1}$ & $0.36 \pm 0.29$ & $0.3 \pm 0.29$ & $0.34 \pm 0.19$ & $0.29 \pm 0.04$ & $0.23 \pm 0.06^{\S}$ & $0.29 \pm 0.07$ \\
\hline Phasic GG-EMG activity $\% \max$ & $1.9 \pm 2.4$ & $2.1 \pm 4.9$ & $2.2 \pm 2.9$ & $1.9 \pm 1.9$ & $4.6 \pm 7.9$ & $2.1 \pm 4.9$ \\
\hline Tonic GG-EMG activity \% max & $1.2 \pm 2.6$ & $0.7 \pm 2.2$ & $1.5 \pm 4.0$ & $0.8 \pm 3.8$ & $2.4 \pm 6.3$ & $0.64 \pm 2.2$ \\
\hline Peak GG-EMG activity $\%$ max & $3.1 \pm 4.8$ & $2.7 \pm 5.9$ & $3.6 \pm 6.7$ & $2.7 \pm 5.1$ & $6.1 \pm 11.6$ & $2.7 \pm 5.9$ \\
\hline
\end{tabular}

Data are presented as mean $\pm \mathrm{SD} . V^{\prime} \mathrm{E}$ : minute ventilation; $V \mathrm{~T}$ : tidal volume; GG-EMG: genioglossus electromyogram. ${ }^{*}:$ measured as time taken for inspiration ( $t$ )/total time of respiratory cycle; ${ }^{\bullet}$ : measured as $V_{T} / t$. ${ }^{*}: p<0.05$ versus placebo treatment, sleep values; ${ }^{+}: p<0.05$ versus baseline, same study day, ${ }^{\S}: p<0.1$ versus placebo treatment, sleep values. 
TABLE 2 Negative pressure pulse data collected during wakefulness

\begin{tabular}{|c|c|c|c|c|}
\hline Pressure reflex and stimulus characteristics & Pre-pentobarbital & Post-pentobarbital & Pre-placebo & Post-placebo \\
\hline Excitation peak amplitude \% baseline & $224 \pm 30$ & $227 \pm 34$ & $236 \pm 34$ & $242 \pm 28$ \\
\hline Minimum mask pressure $\mathrm{cmH}_{2} \mathrm{O}$ & $-16 \pm 2$ & $-17 \pm 2$ & $-19 \pm 2$ & $-17 \pm 1$ \\
\hline Number of artefact-free pulse presentations & $39 \pm 3$ & $35 \pm 3$ & $41 \pm 2$ & $34 \pm 3$ \\
\hline
\end{tabular}

Data are presented as mean \pm SEM. There were no significant differences between conditions for genioglossus reflex characteristics or stimulus magnitudes. $\mathrm{n}=10$

Genioglossus function and peak airflow measured at the same time after starting the negative pressure ramp as at the placebo night

We analysed genioglossus activity and peak airflow at a standardised time $(260 \pm 100 \mathrm{~s}$ after onset of negative pressure drop), defined as the lowest level of mask pressure (at $-5.45 \pm 2.78 \mathrm{cmH}_{2} \mathrm{O}$ ) that we were able to apply under both placebo and pentobarbital conditions. Both flow-limited and no flow-limited breaths were included in this analysis. Phasic genioglossus activity was significantly higher $(3.9 \pm 6.6 \%$ versus $1.3 \pm 1.96 \%$ of maximum activation; $p=0.08$ ), but tonic genioglossus activity $(0.68 \pm 1.2 \%$ versus $0.49 \pm 0.85 \%$ of maximum; $\mathrm{p}=0.3)$ and peak inspiratory airflow $(0.33 \pm 0.1$ versus $\left.0.3 \pm 0.13 \mathrm{~L} \cdot \mathrm{s}^{-1} ; \mathrm{p}=0.17\right)$ did not differ between groups.

Upstream resistance (mask pressure $(P$ mask)/peak airflow $(V \max ))$ taken at the same time tended to be lower under pentobarbital $\left(19 \pm 14 \mathrm{cmH}_{2} \mathrm{O} \cdot \mathrm{L}^{-1} \cdot \mathrm{s}\right)$ compared with placebo $\left(24 \pm 19 \mathrm{cmH}_{2} \mathrm{O} \cdot \mathrm{L}^{-1} \cdot \mathrm{s}(\mathrm{p}=0.066)\right.$.

\section{Respiratory response to $\mathrm{CO}_{2}$}

In one pentobarbital trial, and in two placebo trials, awakening from sleep was observed before steady state hypercapnic stimulation could be achieved. Measurements of the respiratory response to $\mathrm{CO}_{2}$ are therefore reported from nine volunteers.

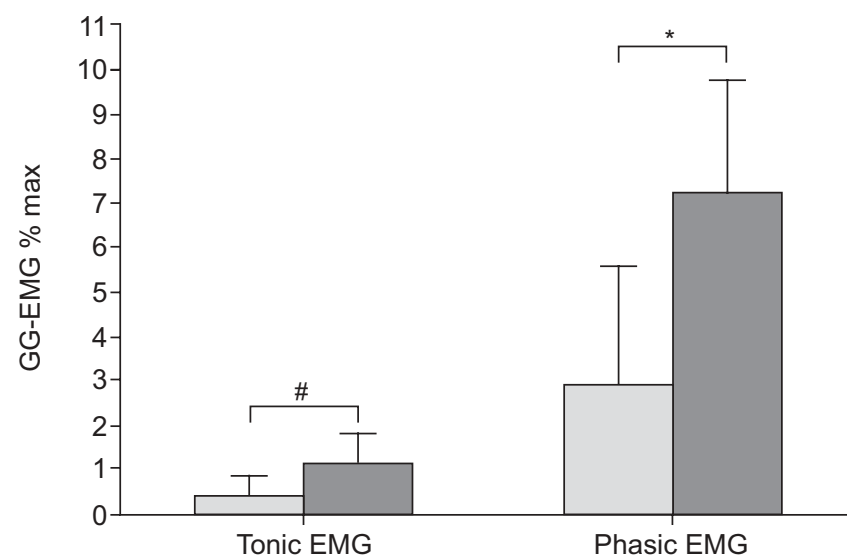

FIGURE 3. Effect of pentobarbital ( $\square$ ) and placebo ( $\square$ ) on genioglossus activity during negative pharyngeal pressure challenges. Average values of genioglossus (GG) electromyogram (EMG) just prior to arousal are shown. Phasic genioglossus activity was significantly higher after pentobarbital $(100 \mathrm{mg})$ compared with the control night, and tonic genioglossus activity tended to be higher. \% max: \% maximal. *: $p<0.05$ versus placebo; ${ }^{*}: p<0.1$ versus placebo.
Increased inspired $\mathrm{CO}_{2}$ augmented the GG-EMG, and the amplitude of this effect was significantly dependent on drug (pentobarbital $>$ placebo) and state (phasic $>$ tonic; table 3). Administration of $\mathrm{CO}_{2}$ significantly increased upper airway resistance by $145 \pm 18 \%$ (placebo) and $147 \pm 17 \%$ (pentobarbital). Flow rate measured at an end-tidal $\mathrm{CO}_{2} 10 \mathrm{mmHg}$ above baseline was not significantly different between groups $(198 \pm 76 \%$ of baseline for placebo versus $196 \pm 68 \%$ for pentobarbital).

\section{DISCUSSION}

Our study found that in healthy volunteers, pentobarbital (100 mg orally) had no effect on respiratory function during wakefulness and did not impair genioglossus muscle function during the awake or sleep states. During stage II sleep, pentobarbital had a mild respiratory depressant effect, manifested as a decrease in peak inspiratory flow, and a rise in end-tidal $\mathrm{CO}_{2}$ and upper airway resistance. However, the hypercapnic responsiveness of the genioglossus muscle improved. Active Pcrit did not significantly change following pentobarbital. However, lower Pcrit values were associated with increased tonic GG-EMG, suggesting clinical relevance of the observed muscle recruitment, i.e. that it was mechanically effective. These results confirm and extend those of Younes et al. [11] as well as our own preclinical studies in rats [16].

An interesting finding of our study is that in humans, pentobarbital increased time to arousal and genioglossus activation preceding arousal. This particular constellation of effects could be useful for OSA patients with low arousal thresholds, ventilatory control instability, or both. YOUNES et al. [3] as well as WeLLMAN et al. [23] have suggested that ventilatory control instability can contribute to the pathogenesis of OSA. Concomitant increases in phasic genioglossus activity and time to arousal should help stabilise breathing patterns by allowing the necessary physiological responses to obstructive events to stabilise the airway, without producing arousals and subsequent ventilatory overshoot that cause ventilatory oscillation in susceptible patients. In support of this idea, YOuNES et al. [24] observed that even patients with severe sleep apnoea have some periods of stable breathing, and we have recently observed that these stable breathing periods are associated with high levels of upper airway dilator muscle activity [25], suggesting that these muscles are necessary and sufficient to protect pharyngeal patency when adequate respiratory stimulation is present for sufficient duration. However, delaying arousal is theoretically deleterious for patients with a high arousal threshold in whom substantial hypoxaemia and hypercapnia could develop. 


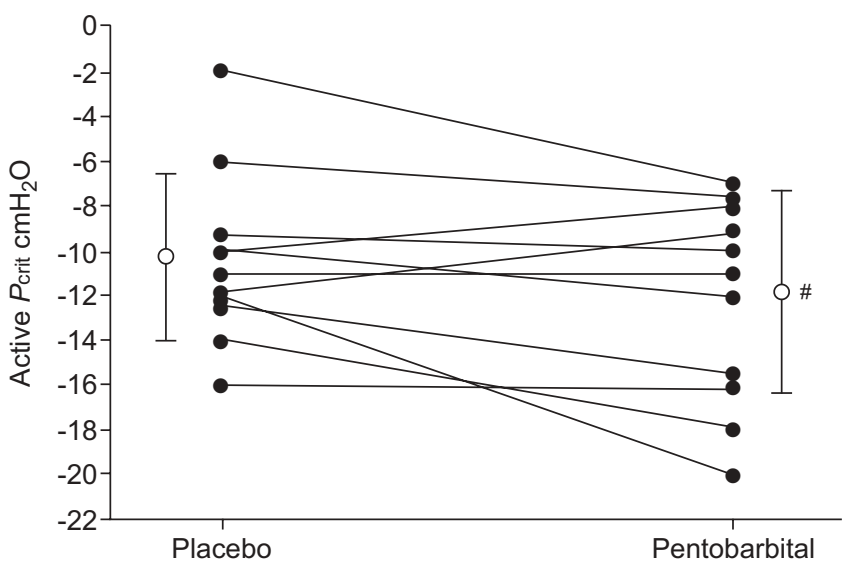

FIGURE 4. Effect of pentobarbital on active upper airway critical closing pressure $\left(P_{\text {crit }}\right)$ during sleep. The figure shows the average $P_{\text {crit }}$ values in 11 subjects during the pentobarbital night compared with the placebo night. Vertical bars represent mean \pm SD. Note that $P$ crit tended to be more negative during the pentobarbital night compared with the placebo night. ${ }^{*}: p=0.11$ versus placebo.

In our study, upper airway closing pressure was stable at $100 \mathrm{mg}$ pentobarbital, a dose that promotes sleep in humans $[26,27]$, without affecting normal breathing or the ventilatory response to $\mathrm{CO}_{2}$ [28]. However, pentobarbital increased upper airway resistance during sleep, leading to decreased peak inspiratory flow rate and mild hypercapnia. In theory, an elevated upper airway resistance may actually be beneficial in those with unstable ventilatory control [29], if the accumulation of respiratory stimuli allows important recruitment of upper airway muscle activity $[15,30]$.

Interestingly, respiratory depression may contribute to upper airway-stabilising effects of pentobarbital. We previously observed a dissociation of pentobarbital's respiratory effects on the genioglossus activation and breathing (inhibition of diaphragmatic activity and consequent hypercapnia) of rats [16]. We therefore speculate that hypercapnia plays a role in

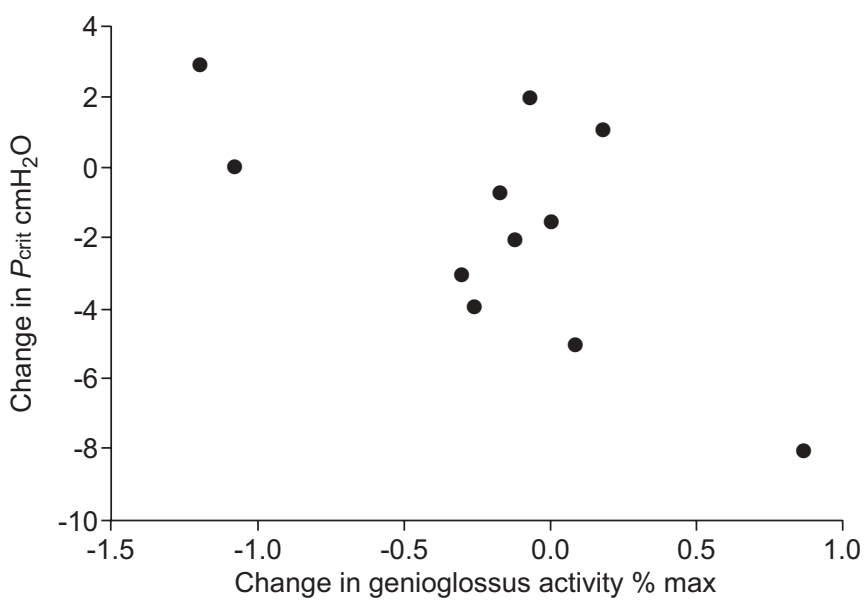

FIGURE 5. Difference in active upper airway critical closing pressure ( $P_{\text {crit }}$ during the pentobarbital night and control night versus the difference in tonic genioglossus activity (as \% of maximum (max) value). Measurements during negative pharyngeal pressure challenges during sleep. Average values from all pressure drops. $r=-0.66, p=0.03$.

mediating pentobarbital's activating effects on the genioglossus, which we observed at the time of arousal [31, 32]. To the extent that chemoreflex activation of the genioglossus muscle may occur independently of ventilatory drive, it is possible that elevated $\mathrm{CO}_{2}$ may partially account for the increase in genioglossus activity observed in parallel with decreased ventilatory drive [31]. Another possibility is that, when negative effort dependence is present, flow may actually improve with reduced ventilatory drive. However, pentobarbital's narrow therapeutic index makes pentobarbital a problematic candidate agent for use as a treatment for OSA. Further work is required to determine whether a pharmacological approach to sleep apnoea therapy is viable using either different agents or by carefully selecting patients for treatment.

An increase in genioglossus activity, as has been observed in our volunteers during the pentobarbital night prior to arousal from

TABLE 3 Respiratory effects of evoked hypercapnia during sleep without continuous positive airway pressure (CPAP)

\begin{tabular}{|c|c|c|c|c|c|c|}
\hline & \multicolumn{2}{|c|}{ Room air } & \multicolumn{2}{|c|}{ PET,CO2 $5 \mathrm{mmHg}$ above baseline } & \multicolumn{2}{|c|}{ PET,CO2 $10 \mathrm{mmHg}$ above baseline } \\
\hline & Pentobarbital & Placebo & Pentobarbital & Placebo & Pentobarbital & Placebo \\
\hline$V^{\prime} E L \cdot \min ^{-1}$ & $6.0 \pm 2.3$ & $5.3 \pm 1$ & $9.6 \pm 3.8$ & $7.9 \pm 2.5$ & $12.1 \pm 6.5^{\S}$ & $12.1 \pm 3.5^{\S}$ \\
\hline VT L. $\min ^{-1}$ & $0.41 \pm 0.15$ & $0.37 \pm 0.07$ & $0.61 \pm 0.19$ & $0.53 \pm 0.17$ & $0.87 \pm 0.33^{\S}$ & $0.79 \pm 2.4^{\S}$ \\
\hline Duty cycle ${ }^{\#}$ & $0.43 \pm 0.13$ & $0.41 \pm 0.04$ & $0.44 \pm 0.23$ & $0.42 \pm 0.05$ & $0.50 \pm 0.30$ & $0.42 \pm 0.03$ \\
\hline Phasic GG-EMG activity $\% \max$ & $4.6 \pm 7.9$ & $2.1 \pm 4.9$ & $5.8 \pm 9.9$ & $2.1 \pm 1.9$ & $14 \pm 24^{f . \# \#}$ & $3.3 \pm 7.7$ \\
\hline Tonic GG-EMG activity $\% \max$ & $2.4 \pm 6.3$ & $0.64 \pm 2.2$ & $3.8 \pm 10.4$ & $0.8 \pm 3.8$ & $5.2 \pm 13.8^{\# \#}$ & $1.0 \pm 3.4$ \\
\hline
\end{tabular}

Data are presented as mean \pm SD. PET,CO2: end-tidal carbon dioxide tension; $V^{\prime} E$ : minute ventilation; VT: tidal volume; GG-EMG: genionglossus electromyogram. \#: measured as time taken for inspiration (tI)/total time of respiratory cycle; ": measured as $V_{T} / t I_{1}{ }^{+}: p<0.05$ for drug effect, i.e., higher GG-EMG during pentobarbital versus placebo (all GG-EMG data during carbon dioxide insufflation, general linear model (mixed model)), s. $p<0.05$ versus placebo treatment (Wilcoxon); ${ }^{f}: p<0.05$ versus baseline, same study day; ${ }^{\# \#: ~} p<0.1$ versus placebo treatment. 
sleep, does not necessarily translate to mechanical improvement. Recently we have observed that pharmacologically evoked genioglossus muscle weakness (partial neuromuscular transmission block) explains only $20 \%$ of the variance of the evoked increase in upper airway closing pressure [33]. Moreover, OSA patients may even have significantly greater basal genioglossal activity compared to controls during wakefulness [20]. Other nonmuscular factors, such as decreases in lung volume [34-36], and fluid displacement into nuchal and peripharyngeal soft tissues [37], could contribute to narrowing and increased airflow resistance of the pharynx, and predispose to pharyngeal collapse in humans.

In the present study, upper airway resistance increased during sleep, and the magnitude of the effect was higher when pentobarbital was given. The respiratory duty cycle was significantly increased by pentobarbital, suggesting that higher resistance was partially offset by an increase in inspiratory time. Our data cannot explain why upper airway resistance during normal breathing was increased, while genioglossal mechano- and chemoresponses were normal or even augmented. Upper airway resistance is influenced by a variety of mechanisms including airflow pattern, mandibular and body position, respiratory timing, as well as end-expiratory lung volume. We speculate that pentobarbital's deleterious effects on upper airway resistance might in part be explained by its reduction of lung volume. In pentobarbital-anaesthetised dogs, phasic electrical activity increases over time in the expiratory muscles, whereas electrical activity of the inspiratory muscles is unchanged [38], which might decrease end-expiratory lung volume.

In our study, the pentobarbital-induced change in tonic but not phasic genioglossus activity correlated with improved Pcrit values. Tonic upper airway muscle activity is critical for maintenance of airway patency [39]. The impact of tonic genioglossus activity on airway patency in OSA patients has been emphasised in a recent study reported from our laboratory [40]. In OSA, reductions in tonic genioglossus activity during rapid eye movement are associated with hypopnoea events and therefore have been suggested to contribute to the higher severity of OSA in that stage [40]. In addition, our recent research in single motor units in the genioglossus has highlighted the importance of tonic motor neurons in affecting overall genioglossal activity and airway mechanics [41]. Ostensibly, the genioglossus phasic activity may be more mechanically effective when influencing a stiffened airway.

\section{Limitations}

We made a comparison between pentobarbital and placebo. Accordingly, our data cannot address whether the observed effects are specific to pentobarbital or a class effect of barbiturates or even $\gamma$-aminobutyric acid A agonists.

The increases in GG-EMG at the end of the negative pressure ramp may be secondary to the prolonged latency to arousal (with lower mask and pharyngeal pressures and an increase in $\mathrm{CO}_{2}$ ) and increased resistance, but may also include a specific barbiturate stimulatory effect. The greater increase in the genioglossus response to evoked hypercapnia during the pentobarbital compared with placebo may provide some evidence for a primary stimulatory effect of the barbiturate on genioglossus EMG. Moreover, genioglossus activity measured at a standardized time after onset of a pressure ramp, revealed higher values of phasic genioglossus activity during the pentobarbital compared with placebo, suggesting that barbiturates may have some stimulatory effects on genioglossus muscle.

At the end of the pressure ramps, the volunteers showed considerable flow-limited breathing (even at a fixed flow of $0.2 \mathrm{~L} \cdot \mathrm{s}^{-1}$ ), which complicates resistance determinations. As the pharyngeal tissues collapse, the epiglottic pressure is no longer the downstream pressure, and thus airflow resistance is not simply a function of the pressure drop from the mask to the epiglottic catheter. While we have included peak-flow during the ramps, we do not know an ideal way of reporting the resistance at the end of the pressure ramps during flow limitation.

We believe more OSA research should address arousability, a variable that is associated with sleepiness and may have therapeutic implications [42]. We assessed arousability by the arousal response time during step reduction of the airway pressures. During OSA, arousal could be caused by various factors, such as hypercapnia, hypoxaemia, intrathoracic pressure, and by interaction among these. The arousal response examined in this study is one aspect of the responses in normal subjects and may not reflect arousablity during OSA.

In summary, in healthy volunteers given pentobarbital, time to arousal and phasic genioglossus activity immediately prior to arousal were increased and genioglossus reflex activation was maintained. However, pentobarbital increased upper airway resistance during sleep leading to decreased peak flow and mild hypercapnia while active upper airway closing pressure was stable. These findings make it difficult to predict whether or not manipulating arousal threshold with pentobarbital may be a viable therapeutic strategy for subsets of sleep apnoea patients. Its narrow therapeutic index makes pentobarbital a problematic candidate agent. Further work will be required to determine whether a pharmacological approach to sleep apnoea therapy is viable using either different agents or by carefully selecting patients for treatment.

\section{SUPPORT STATEMENT}

This work was supported by grants from the National Institute of Health (NIH) (P50 HL060292, RO1-HL73146, AG024837, R01HL085188, AHA 0840159N, NIH R01 HL090897, K24 HL-093218), and a grant from the German Research Council (DFG-EI684/2-1). D.J. Eckert is supported by an Overseas Based Biomedical Fellowship from the National Health and Medical Research Council of Australia (510392).

\section{STATEMENT OF INTEREST}

A statement of interest for A. Malhotra can be found at www.erj. ersjournals.com $/ \mathrm{misc} /$ statements.dtl

\section{REFERENCES}

1 Young T, Palta M, Dempsey J, et al. The occurrence of sleepdisordered breathing among middle-aged adults. New Engl J Med 1993; 32: 1230-1235.

2 Isono S. Obstructive sleep apnea of obese adults: pathophysiology and perioperative airway management. Anesthesiology 2009; 110: 908-921. 
3 Younes M. Role of arousals in the pathogenesis of obstructive sleep apnea. Am J Respir Crit Care Med 2004; 169: 623-633.

4 Eckert DJ, Malhotra A, Jordan AS. Mechanisms of apnea. Prog Cardiovasc Dis 2009; 51: 313-323.

5 Berry RB, Gleeson K. Respiratory arousal from sleep: mechanisms and significance. Sleep 1997; 20: 654-675.

6 Longobardo GS, Evangelisti CJ, Cherniack NS. Analysis of the interplay between neurochemical control of respiration and upper airway mechanics producing upper airway obstruction during sleep in humans. Exp Physiol 2008; 93: 271-287.

7 Wellman A, Malhotra A, Jordan AS, et al. Chemical control stability in the elderly. J Physiol 2007; 581: 291-298.

8 Carley DW, Olopade C, Ruigt GS, et al. Efficacy of mirtazapine in obstructive sleep apnea syndrome. Sleep 2007; 30: 35-41.

9 Carley DW, Radulovacki M. Mirtazapine, a mixed-profile serotonin agonist/antagonist, suppresses sleep apnea in the rat. Am J Respir Crit Care Med 1999; 160: 1824-1829.

10 Heinzer RC, White DP, Jordan AS, et al. Trazodone increases arousal threshold in obstructive sleep apnoea. Eur Respir J 2008; 31: 1308-1312.

11 Younes M, Park E, Horner RL. Pentobarbital sedation increases genioglossus respiratory activity in sleeping rats. Sleep 2007; 30: 478-488.

12 Berry RB, Kouchi K, Bower J, et al. Triazolam in patients with obstructive sleep apnea. Am J Respir Crit Care Med 1995; 151: 450-454.

13 Berry RB, Bonnet MH, Light RW. Effect of ethanol on the arousal response to airway occlusion during sleep in normal subjects. Am Rev Respir Dis 1992; 145: 445-452.

14 Veasey SC, Fenik P, Panckeri K, et al. The effects of trazodone with L-tryptophan on sleep-disordered breathing in the English bulldog. Am J Respir Crit Care Med 1999; 160: 1659-1667.

15 Stanchina ML, Malhotra A, Fogel RB, et al. Genioglossus muscle responsiveness to chemical and mechanical stimuli during nonrapid eye movement sleep. Am J Respir Crit Care Med 2002; 165 : 945-949.

16 Eikermann M FP, Zaremba S, Jordan AS, et al. Pentobarbital dosedependently increases respiratory genioglossus muscle activity while impairing diaphragmatic function in anesthetized rats. Anesthesiology 2009; 110: 1327-1334.

17 Eckert DJ, McEvoy RD, George KE, et al. Genioglossus reflex inhibition to upper airway negative-pressure stimuli during wakefulness and sleep in healthy males. J Physiol 2007; 581: 1193-1205.

18 Eckert DJ, McEvoy RD, George KE, et al. Effects of hypoxia on genioglossus and scalene reflex responses to brief pulses of negative upper airway pressure during wakefulness and sleep in healthy men. J Appl Physiol 2008; 104: 1426-1435.

19 Rechtschaffen A, Kales A, eds. A Manual of Standarized Terminology, Techniques and Scoring System for Sleep Stages of Human Subjects. Bethesda, US Government Printing Office, 1968.

20 Mezzanotte WS, Tangel DJ, White DP. Waking genioglossal electromyogram in sleep apnea patients versus normal controls (a neuromuscular compensatory mechanism). J Clin Invest 1992; 89: 1571-1579.

21 Patil SP, Schneider H, Marx JJ, et al. Neuromechanical control of upper airway patency during sleep. J Appl Physiol 2007; 102: 547-556.

22 Pierce R, White D, Malhotra A, et al. Upper airway collapsibility, dilator muscle activation and resistance in sleep apnoea. Eur Respir J 2007; 30: 345-353.
23 Wellman A, Jordan AS, Malhotra A, et al. Ventilatory control and airway anatomy in obstructive sleep apnea. Am J Respir Crit Care Med 2004; 170: 1225-1232.

24 Younes M. Contributions of upper airway mechanics and control mechanisms to severity of obstructive apnea. Am J Respir Crit Care Med 2003; 168: 645-658.

25 Jordan AS, Wellman A, Heinzer RC, et al. Mechanisms used to restore ventilation after partial upper airway collapse during sleep in humans. Thorax 2007; 62: 861-867.

26 Kay DC, Jasinski DR, Eisenstein RB, et al. Quantified human sleep after pentobarbital. Clin Pharmacol Ther 1972; 13: 221-231.

27 Kales A, Kales JD, Jacobson A, et al. Effects of methyprylon and pentobarbital on sleep patterns. Electroencephalogr Clin Neurophysiol 1968; 24: 397.

28 Johnstone RE, Lief PL, Kulp RA, et al. Combination of $\Delta 9$ tetrahydrocannabinol with oxymorphone or pentobarbital: effects on ventilatory control and cardiovascular dynamics. Anesthesiology 1975; 42: 674-684.

29 Dempsey JA. Crossing the apnoeic threshold: causes and consequences. Exp Physiol 2005; 90: 13-24.

30 Saboisky JP, Chamberlin NL, Malhotra A. Potential therapeutic targets in obstructive sleep apnoea. Expert Opin Ther Targets 2009; 13: 795-809.

31 Horner RL, Liu X, Gill H, et al. Effects of sleep-wake state on the genioglossus versus diaphragm muscle response to $\mathrm{CO}_{2}$ in rats. J Appl Physiol 2002; 92: 878-887.

32 Bailey EF, Jones CL, Reeder JC, et al. Effect of pulmonary stretch receptor feedback and $\mathrm{CO}_{2}$ on upper airway and respiratory pump muscle activity in the rat. J Physiol 2001; 532: 525-534.

33 Herbstreit F, Peters J, Eikermann M. Impaired upper airway integrity by residual neuromuscular blockade: increased airway collapsibility and blunted genioglossus muscle activity in response to negative pharyngeal pressure. Anesthesiology 2009; 110: 1253-1260.

34 Heinzer RC, Stanchina ML, Malhotra A, et al. Lung volume and continuous positive airway pressure requirements in obstructive sleep apnea. Am J Respir Crit Care Med 2005; 172: 114-117.

35 Heinzer RC, Stanchina ML, Malhotra A, et al. Effect of increased lung volume on sleep disordered breathing in sleep apnoea patients. Thorax 2006; 61: 435-439.

36 Van de Graaff WB. Thoracic influence on upper airway patency. J Appl Physiol 1988; 65: 2124-2131.

37 Chiu KL, Ryan CM, Shiota S, et al. Fluid shift by lower body positive pressure increases pharyngeal resistance in healthy subjects. Am J Respir Crit Care Med 2006; 174: 1378-1383.

38 Warner DO, Joyner MJ, Rehder K. Electrical activation of expiratory muscles increases with time in pentobarbital-anesthetized dogs. J Appl Physiol 1992; 72: 2285-2291.

39 Tangel DJ, Mezzanotte WS, Sandberg EJ, et al. Influences of NREM sleep on the activity of tonic versus inspiratory phasic muscles in normal men. J Appl Physiol 1992; 73: 1058-1066.

40 Jordan AS, White DP, Lo YL, et al. Airway dilator muscle activity and lung volume during stable breathing in obstructive sleep apnea. Sleep 2009; 32: 361-368.

41 Wilkinson V, Malhotra A, Nicholas CL, et al. Discharge patterns of human genioglossus motor units during sleep onset. Sleep 2008; 31: 525-533.

42 Masa JF, Corral J, Teran J, et al. Apnoeic and obstructive nonapnoeic sleep respiratory events. Eur Respir J 2009; 34: 156-161. 